\title{
MODELING THE TRANSFER FUNCTION FOR THE DARK ENERGY SURVEY
}

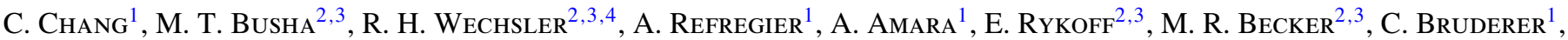

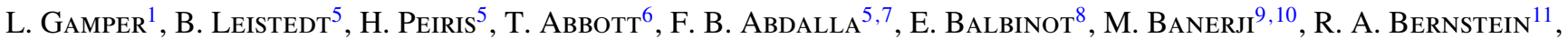 \\ E. Bertin ${ }^{12}$, D. Brooks ${ }^{5}$, A. Carnero ${ }^{13,14}$, S. Desai ${ }^{15,16}$, L. N. DA Costa ${ }^{13,14}$, C. E Cunha ${ }^{17}$, T. Eifler $^{18}$, \\ A. E. Evrard ${ }^{12,19,20}$, A. Fausti Neto ${ }^{14}$, D. Gerdes ${ }^{19}$, D. Gruen ${ }^{21,22}$, D. JAmes ${ }^{6}$, K. KuehN ${ }^{23}$, M. A. G. Maia ${ }^{13,14}$, \\ M. Makler ${ }^{24}$, R. Ogando ${ }^{13,14}$, A. Plazas ${ }^{25}$, E. SancheZ ${ }^{26}$, B. Santiago ${ }^{14,27}$, M. Schubnell ${ }^{19}$, I. SEvilla-Noarbe $^{26}$, \\ C. Smith $^{6}$, M. Soares-Santos ${ }^{28}$, E. Suchyta ${ }^{29}$, M. E. C. Swanson ${ }^{30}$, G. TARle ${ }^{19}$, And J. Zuntz ${ }^{31}$ \\ ${ }^{1}$ Department of Physics, ETH Zurich, Wolfgang-Pauli-Strasse 16, CH-8093 Zurich, Switzerland \\ ${ }^{2}$ Kavli Institute for Particle Astrophysics and Cosmology, P.O. Box 2450, Stanford, CA 94305, USA \\ ${ }^{3}$ SLAC National Accelerator Laboratory, 2575 Sand Hill Road, Menlo Park, CA 94025, USA \\ ${ }^{4}$ Department of Physics, Stanford University, 382 Via Pueblo Mall, Stanford, CA 94305, USA \\ ${ }^{5}$ Department of Physics and Astronomy, University College London, London WC1E 6BT, UK \\ ${ }^{6}$ Cerro Tololo Inter-American Observatory, National Optical Astronomy Observatory, Casilla 603, La Serena, Chile \\ ${ }^{7}$ Department of Physics and Electronics, Rhodes University, P.O. Box 94, Grahamstown 6140, South Africa \\ 8 Department of Physics, University of Surrey, Guildford GU2 7XH, UK \\ ${ }^{9}$ Institute of Astronomy, University of Cambridge, Madingley Road, Cambridge CB3 0HA, UK \\ ${ }^{10}$ Kavli Institute for Cosmology, University of Cambridge, Madingley Road, Cambridge CB3 OHA, UK \\ ${ }^{11}$ Carnegie Observatories, 813 Santa Barbara St., Pasadena, CA 91101, USA \\ 12 Institut d'Astrophysique de Paris, Univ. Pierre et Marie Curie and CNRS UMR7095, F-75014 Paris, France \\ ${ }^{13}$ Observatório Nacional, Rua Gal. José Cristino 77, Rio de Janeiro, RJ 20921-400, Brazil \\ ${ }^{14}$ Laboratório Interinstitucional de e-Astronomia-LIneA, Rua Gal. José Cristino 77, Rio de Janeiro, RJ 20921-400, Brazil \\ ${ }^{15}$ Department of Physics, Ludwig-Maximilians-Universität, Scheinerstr. 1, D-81679 Munich, Germany \\ ${ }^{16}$ Excellence Cluster Universe, Boltzmannstr. 2, D-85748 Garching, Germany \\ ${ }_{17}$ Robert Bosch LLC, 4009 Miranda Ave, Suite 225, Palo Alto, CA 94304, USA \\ 18 Jet Propulsion Laboratory, California Institute of Technology, 4800 Oak Grove Dr., Pasadena, CA 91109, USA \\ ${ }^{19}$ Department of Physics, University of Michigan, Ann Arbor, MI 48109, USA \\ ${ }^{20}$ Department of Astronomy, University of Michigan, Ann Arbor, MI 48109, USA \\ ${ }^{21}$ University Observatory Munich, Scheinerstrasse 1, D-81679 Munich, Germany \\ ${ }^{22}$ Max Planck Institute for Extraterrestrial Physics, Giessenbachstrasse, D-85748 Garching, Germany \\ ${ }^{23}$ Australian Astronomical Observatory, North Ryde, NSW 2113, Australia \\ ${ }^{24}$ ICRA, Centro Brasileiro de Pesquisas Físicas, Rua Dr. Xavier Sigaud 150, CEP 22290-180, Rio de Janeiro, RJ, Brazil \\ ${ }^{25}$ Brookhaven National Laboratory, Bldg 510, Upton, NY 11973, USA \\ ${ }^{26}$ Centro de Investigaciones Energéticas, Medioambientales y Tecnológicas (CIEMAT), Madrid, Spain \\ ${ }^{27}$ Instituto de Física, Universidade Federal do Rio Grande do Sul, Av. Bento Gonçalves, 9500, Porto Alegre, RS 91501-970, Brazil \\ ${ }^{28}$ Fermi National Accelerator Laboratory, P.O. Box 500, Batavia, IL 60510, USA \\ ${ }^{29}$ Center for Cosmology and Astro-Particle Physics, The Ohio State University, Columbus, OH 43210, USA \\ ${ }^{30}$ National Center for Supercomputing Applications, 1205 West Clark St., Urbana, IL 61801, USA \\ 31 Jodrell Bank Center for Astrophysics, School of Physics and Astronomy, University of Manchester, Oxford Road, Manchester M13 9PL, UK \\ Received 2014 October 30; accepted 2015 January 8; published 2015 March 4
}

\begin{abstract}
We present a forward-modeling simulation framework designed to model the data products from the Dark Energy Survey (DES). This forward-model process can be thought of as a transfer function-a mapping from cosmological/ astronomical signals to the final data products used by the scientists. Using output from the cosmological simulations (the Blind Cosmology Challenge), we generate simulated images (the Ultra Fast Image Simulator) and catalogs representative of the DES data. In this work we demonstrate the framework by simulating the $244 \mathrm{deg}^{2} \mathrm{coadd}^{2}$ images and catalogs in five bands for the DES Science Verification data. The simulation output is compared with the corresponding data to show that major characteristics of the images and catalogs can be captured. We also point out several directions of future improvements. Two practical examples-star-galaxy classification and proximity effects on object detection - are then used to illustrate how one can use the simulations to address systematics issues in data analysis. With clear understanding of the simplifications in our model, we show that one can use the simulations side-by-side with data products to interpret the measurements. This forward modeling approach is generally applicable for other upcoming and future surveys. It provides a powerful tool for systematics studies that is sufficiently realistic and highly controllable.
\end{abstract}

Key words: methods: data analysis - methods: numerical - surveys - techniques: image processing

\section{INTRODUCTION}

We have entered an exciting era of optical surveys. In recent years, the Kilo Degree Survey ${ }^{32}$ (KiDS; de Jong et al. 2013), the Panoramic Survey Telescope and Rapid Response System ${ }^{33}$

\footnotetext{
32 http://kids.strw.leidenuniv.nl/

33 http://pan-starrs.ifa.hawaii.edu/public/
}

(Pan-STARRS; Hodapp et al. 2004), the Hyper Suprime-Cam Survey $^{34}$ (HSC; Miyazaki et al. 2012), and the Dark Energy Survey ${ }^{35}$ (DES; The Dark Energy Survey Collaboration 2005) have all started to take data. In particular, DES will cover the widest area (one-eighth of the sky), and the resulting enormous

\footnotetext{
${ }^{34}$ http://www.naoj.org/Projects/HSC/

$35 \mathrm{http}: / /$ www.darkenergysurvey.org/
} 
data sets will allow one to achieve very high statistical precision in measuring cosmological parameters. We will soon be able to test with multiple cosmological probes the standard $\Lambda \mathrm{CDM}$ cosmological model, and gain a better understanding of the nature of dark energy (Albrecht et al. 2006; Frieman et al. 2008; Huterer 2010; Allen et al. 2011; Weinberg et al. 2013; Ruiz-Lapuente 2014).

As the statistical uncertainties are reduced by orders of magnitude in these large data sets, various systematic uncertainties in analyzing the data become important (Huterer et al. 2006; Amara \& Réfrégier 2008; Ho et al. 2013; Agarwal et al. 2014; Scolnic et al. 2014). Different cosmological probes are sensitive to different systematic effects. However, generally, as all measurements begin from the same processed images and catalogs, the first-order systematic effects in these data products need to be well understood. In other words, one needs to understand how the information coming from the sky is transformed into the processed images and catalogs on which we base our scientific measurements. Moreover, one needs to understand how this transformation depends on the properties of the astronomical sources and the observing conditions. This paper seeks to understand this complicated process - the "transfer function"- - for DES via forward-modeling. The goal of this work is to model the coadd images and the catalogs from DES. Although this framework still contains several simplifications (see Section 3.1), it is the necessary first step in building a fully realistic simulation pipeline. Note also that although we focus on DES in this paper, our methodology is generally applicable for all upcoming and future large surveys.

The concept of modeling the transfer function for a specific experiment has a long history in the field of particle physics (Bengtsson \& Sjöstrand 1987; Nelson \& Namito 1990; Marchesini et al. 1992; Agostinelli et al. 2003; Binder \& Heermann 2010; Beringer et al. 2012). In fact, the results of particle physics experiments can only be interpreted in terms of their corresponding Monte Carlo simulations. In optical astronomy, however, the idea of forward-modeling is less mature, despite the fact that highly developed simulation tools exist for individual steps of the transfer function. For example, cosmological simulations such as those by Hilbert et al. (2009), Kiessling et al. (2011), Gerke et al. (2013), Riebe et al. (2013), and White et al. (2014) begin with $N$-body simulations and develop prescriptions for assigning astronomical objects to dark matter halos. Springel \& Hernquist (2003), Smith et al. (2008), and Vogelsberger et al. (2012) use different techniques to simulate various hydrodynamic processes in structure formation and link to observables related to cosmology. Peng et al. (2002) uses simulated galaxy images to help understand the study of galaxy morphology. Bertin (2009), Bridle et al. (2010), Kitching et al. (2012), and Bergé et al. (2013) simulate astronomical images with simple instrumental effects to understand how well one can recover information from noisy data. Finally, Peterson \& Jernigan (2013) focuses on the detail modeling of the astronomical instrument to understand how the instrument design affects the imaging data. Although these different simulations are very helpful for understanding the technical issues in the separate areas, one cannot straightforwardly infer how the results in different parts of the transfer function couple to each other. The recent attempt described in Connolly et al. (2010) is one of the first efforts to consolidate the issue by connecting all types to an end-to-end simulation framework for one specific project, the Large Synoptics Survey Telescope (LSST). Our work is based on the same philosophy, but instead of modeling a future instru- ment like LSST, the aim is to model DES, which is currently taking data.

We extend from the Blind Cosmology Challenge simulations (BCC; Busha et al. 2013) to include processed images from the Ultra Fast Image Generator (UFig; Bergé et al. 2013) and catalog products that come from a similar analysis pipeline as that used in the DES Data Management (DESDM; Ngeow et al. 2006; Sevilla et al. 2011; Desai et al. 2012; Mohr et al. 2012). Our implementation is similar to the earlier DES data challenges described in Lin et al. (2010) and Sevilla et al. (2011), where DES simulations were generated before the existence of data to test data management and science analysis software. This work is complementary to the earlier data challenges in that the simulations in this work is guided by the actual DES data and data processing pipeline being used, which was not available at the time of the data challenge.

This paper is organized as follows. In Section 2, we briefly introduce the DES and the relevant data products that are used in this paper. In Section 3 we describe in detail the forward-modeling framework, including individual simulation and analysis tools, as well as the interfacing between them. A series of quality assurance tests are performed in Section 4 to examine the output products of our framework. We cross-check with early DES data to ensure the output captures the main characteristics of the data. We then demonstrate in Section 5 two practical applications where we use this forward-modeling framework to address specific technical questions in the data analysis process. Finally, we conclude in Section 6.

An example of the simulation output and supporting documentation from this work can be found at http://www.phys.ethz.ch/ ast/cosmo/bcc_ufig_public/.

\section{THE DARK ENERGY SURVEY}

The DES is a wide-field optical survey that officially began in 2013 August (Diehl et al. 2014) and will continue to survey the sky through 2018. The full DES footprint will cover oneeighth of the full sky $\left(5,000 \mathrm{deg}^{2}\right)$ in five optical bands ( $\left.g r i z Y\right)$. The homogeneous wide-field nature of the data set will be important for cosmology studies on very large scales. The primary instrument for DES is a newly assembled wide-field (3 $\left.\mathrm{deg}^{2}\right)$ mosaic camera, the Dark Energy Camera (DECam; Diehl \& Dark Energy Survey Collaboration 2012), installed on the $4 \mathrm{~m}$ Blanco telescope at the Cerro Tololo Inter-American Observatory (CTIO) in Chile.

The raw images taken each night are collected and jointly processed with the DESDM software. In addition to the zeroth-order image processing (flat-fielding, bias correction, de-trending, etc.), the DESDM pipeline contains mainly software packages described in Ngeow et al. (2006), Sevilla et al. (2011), Desai et al. (2012), and Mohr et al. (2012)_SCAMP (astrometry; Bertin 2006), SWARP (image coaddition; Bertin et al. 2002), PSFEx (modeling of the point-spread function (PSF); Bertin 2011), and SEXTRACTOR (object detection and measurement; Bertin \& Arnouts 1996). With continual improvement in the pipeline, DESDM performs regular releases of the data products. The main product from DESDM are images and catalogs of objects with calibrated properties.

The initial pre-season of DES observations were labeled as Science Verification (SV) imaging, which took place from 2012 November-2013 February. These images were processed by the DESDM pipeline version "SVA1" (B. Yanny et al., in preparation) to produce coadd images and SExTRACTOR catalogs. 


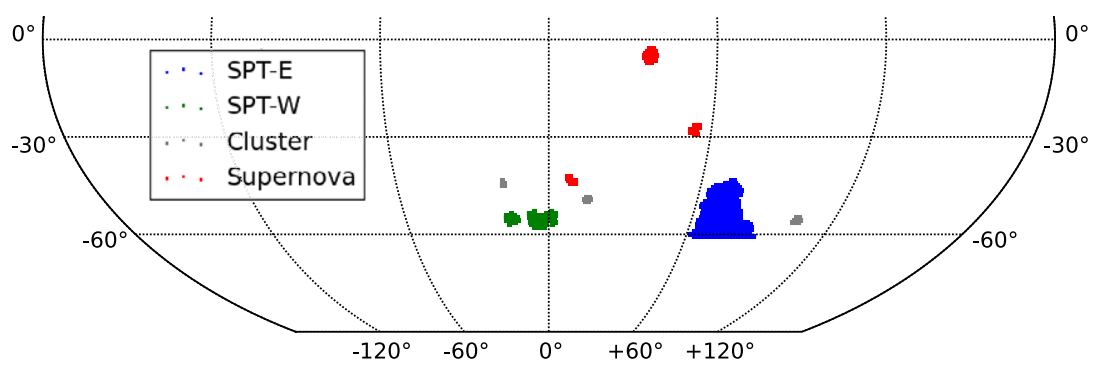

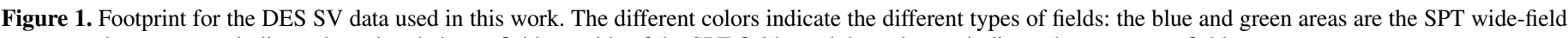
coverage, the gray areas indicate the pointed cluster fields outside of the SPT fields, and the red areas indicate the supernova fields.

Additional quality checks and calibration were performed by DES scientists, which included cropping out bad regions contaminated by satellite and airplane trails, as well as the region at declination $<-61^{\circ}$ which has a very high stellar density due to the presence of the Large Magellanic Cloud (SVA1 Gold; E. Rykoff et al., in preparation). After all cuts, the total sky cov-

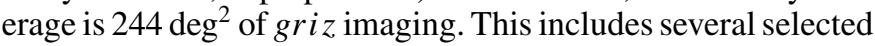
wide fields, pointed cluster fields (RXC J2248.7-4431, 1E 065756, SCSO J233227-535827, and El Gordo), and deep supernova (SN) fields. Figure 1 shows the full SVA1 footprint and how the different fields are distributed. The SN fields are revisited every five to seven days with longer exposures, and are therefore one to two magnitudes deeper than the other fields, particularly in the $i$ and $z$ bands. In this work, we base our forward-modeling framework on the SVA1 Gold catalogs. As the DESDM software and image quality continue to improve for future releases, our modeling framework will adjust accordingly.

\section{FORWARD-MODELING}

In this section we briefly introduce the three major elements of our forward-modeling framework: two simulation tools (Sections 3.2 and 3.3) and the analysis software (Section 3.4). We then describe how the interfaces between the three components are implemented (Section 3.5) and the computational cost (Section 3.6). First, however, we list in Section 3.1 the main simplifications used in this framework.

\subsection{Simplifications}

The current framework as described below contains several simplifications. As we will discuss in Section 6, more sophistication and realism is planned to be added to the framework as required from different science cases. The main simplifications of the current framework are the following: (1) We begin the forward-modeling from coadd images instead of singleexposure images, thus bypassing the process of stacking images. (2) The PSF, airmass, background (limiting magnitude), quantum efficiency, and throughput are constant in each filter with no spatial variation across an image. (3) The background model is simplistic (Gaussian noise plus Lanczos resampling) and does not properly model the correlation of noise in the images. (4) There are no artifacts such as bad/hot columns on the detectors, satellites, cosmic rays, etc.

It is important to stress that the focus of this forward-modeling framework is not to make simulations that are identical to the data (nor is it possible to do so exactly). Rather, it is to capture the important characteristics of the data in a controlled environment where we know the truth. This allows us to interpret the measurements in a clean fashion within the limitations of the simulations. As a result, despite these simplifications, many data-related issues can already be investigated as we demonstrate in Sections 4 and 5. The results from these simplified simulations would also be important for interpreting more realistic simulations in the future as we incorporate more physics in the forward model.

\subsection{The Mock Sky Catalog}

The primary input to our framework is a mock sky catalogs of astronomical sources. In this work, we use the Aadvark v1.0d catalogs generated as part of the BCC. The BCC cata$\log$ generation begins with particle light cones from a series of large (1-4 Gpc hr $\left.{ }^{-1}\right) \mathrm{N}$-body simulations with a defined cosmology (a flat LCDM cosmology in this case). The Adding Density Determined GAlaxies to Lightcone Simulations algorithm (ADDGALS; Busha et al. 2013) associates galaxies to the dark matter particles by using a Sub-Halo Abundance Matching (SHAM) catalog (Conroy et al. 2006; Behroozi et al. 2010) generated from a high resolution, low-volume tuning simulation to determine a probabilistic relation between a galaxy's magnitude and its local dark matter density. The algorithm then assigns basic properties (luminosity, color, etc.) to each galaxy using a training set of spectroscopic data from the Sloan Digital Sky Survey (SDSS) DR6 Value-Added Galaxy Catalog (Blanton et al. 2005) to match simulated galaxies to observed counterparts using the local galaxy environment. The training procedure is performed at low redshift and extrapolated to high redshift so that the color distribution simultaneously matches the photometric data in SDSS DR8 and DEEP2. The intrinsic shape and size of each galaxy is then set to match to observations from the SuprimeCam deep $i^{\prime}$-band data (Dietrich et al. 2012). Finally, the galaxies are lensed by the multipleplane ray-tracing code, Curved-sky grAvitational Lensing for Cosmological Light conE simulatioNS (CALCLENS; Becker 2013) to give perturbed shapes, positions and magnitudes. Additionally, a stellar distribution is added based on the TRIdimentional modeL of thE GALaxy code (Trilegal, Girardi et al. 2012; Balbinot et al. 2012), and the quasar model is based on Maddox et al. (2012). The full details of the BCC catalogs would be described in an upcoming paper.

These BCC catalogs serve as the "true" sky after the sources have been lensed by the large scale structures before the light enters the atmosphere. For this work, the main properties used in the BCC catalogs are the magnitude, size, color, redshift, and shape distributions of objects. The main requirement is that these distributions in the BCC catalog are modeled for objects fainter than the limiting magnitude of the data set we wish to model.

There are several advantages of using such sophisticated cosmological simulations as our input compared to using parametrized star/galaxy distributions (see our earlier work 
in Bergé et al. 2013). First, one preserves the cosmological clustering of the galaxies. Second, one simultaneously retains a self-consistent cosmology among clustering, lensing, and redshift evolution of galaxies. Finally, the correlation between the magnitudes of objects in different filter bands (i.e., colors) are also self-consistent. Note however, that the BCC catalogs cut off at a magnitude only slightly deeper than the DES main survey limiting magnitude. This suggests that the fainter objects that contribute to the background will be missing in our images and we cannot simulate properly the deeper SN fields. One would need to examine the impact of these missing faint objects on the measurement of interest when using the simulations from this framework.

\subsection{The Image Simulation Software}

The Ultra Fast Image Generator (UFIG; for full detail of the implementation of UFIG, see Bergé et al. 2013) is a fast image simulation code that generates scientific astronomical images that capture the major characteristics of a given instrument, as specified by the user. The computational time required for UFIG to generate images in this work is much shorter than the time required to analyze the images (see Section 3.6).

We briefly describe here the image rendering process in UFIG. First, the apparent magnitudes of stars and galaxies are converted into number of photons expected at the focal plane, given the atmosphere and instrumental throughput in the specific filter band. Then, images of the galaxies are generated by drawing probabilistically, one photon at a time, from the galaxy profile model (single Sérsic profile with varying Sérsic index; Sérsic 1963). Next, we construct a model for the PSF given a desired seeing value. The galaxies are then convolved with the PSF model by displacing the photons randomly according to a probability density function described by the PSF profile. The image is then pixelated. Stars are generated directly on the pixels, with the same profile as the PSF model and appropriate Poisson noise on the pixel values. The stars and galaxies are generated via different approaches to optimize the computational speed. These pixel values are then converted into electronic units (ADUs) and an user-specified Gaussian noise is added. Finally, the full image is convolved with a Lanczos filter of size 3 (Duchon 1979) to simulate the correlation of the noise in a coadd image. The full image is then rescaled to a given magnitude zeropoint.

\subsection{The Data Processing Software}

As mentioned in Section 2, the DESDM pipeline uses a suite of software packages to produce the final catalog. Since we simulate the processed coadd images directly from UFIG (Section 3.3), we bypass several steps in the DESDM pipeline. These are simplifications that can be improved upon in the future. The two main packages involved in our framework are PSFEx and SEXTRACTOR.

PSFEx is a software that constructs a model for the PSF of an image. Accurately knowing the PSF is important for later steps in the pipeline such as photometry measurements and galaxy profile-fitting. SExTRACTOR is the main measurement software in the process. It estimates the background, detects objects, and conducts the basic measurements for each object. These include magnitudes estimated with several different approaches, various size estimates, parametrized model of the object profile, and classifiers that help the user identify different types of objects. As the output is sensitive to detailed settings in the PSFEX and
SEXTRACTOR configuration, we match the setting to that used in the SVA1 catalog whenever possible.

\subsection{Bridging Heaven and Earth}

The three basic elements of the forward-modeling framework described above are interfaced and connected as described in the following steps.

\subsubsection{BCC Catalog $\rightarrow$ UFIG Catalog}

The first step involves converting the "sky information" in the BCC catalogs into "image information" that can be used by UFIG. We start by defining pointing positions on the sky, from which we draw a $0.75 \times 0.75 \mathrm{deg}^{2}$ area where the image will be simulated. The image size is defined by that of DESDM coadd images.

The information in the $\mathrm{BCC}$ catalogs is then translated into UFIG internal parameters. Object coordinates are converted into physical positions on the image with the appropriate World Coordinate System (WCS) transformation. All images are linearly projected from the sky with a pixel scale of 0.27 arcsec pixel ${ }^{-1}{ }^{36}$ The apparent magnitude of stars and galaxies, as well as the ellipticity of galaxies are taken directly from the BCC catalogs. The intrinsic galaxy size information is based on the BCC catalogs but adjusted slightly so that the $2 \mathrm{~d}$ distribution in apparent magnitude and intrinsic size is consistent with that derived from the COSMOS data (Jouvel et al. 2009). The adjustment is needed because the BCC catalog takes an approximate approach when converting the observed galaxy size into the intrinsic galaxy size. Finally, the galaxy is modeled by a single Sérsic profile, where the Sérsic indices are band-independent and drawn randomly from the following distributions:

$$
f(n)=0.2+ \begin{cases}\exp (N(0.3,0.5)+N(1.6,0.4)) & \text { if } i<20 \\ \exp (N(0.2,1)) & \text { if } i \geqslant 20\end{cases}
$$

$N(\mu, \sigma)$ denotes a normal distribution of mean $\mu$ and standard deviation $\sigma$. Equation (1) was derived in Bergé et al. (2013) from fitting deep $i$-band images (Griffith et al. 2012). A more sophisticated Sérsic distribution that also takes into account the band dependencies would be a direction for future improvement. The Sérsic index is the only parameter of the source properties external to the BCC catalogs.

\subsubsection{UFIG Catalog $\rightarrow$ UFIG Image}

Next, we simulate a UFIG image from the source catalog generated from the previous step. The instrument characteristics and observing conditions need to be specified for each image. These parameters include the throughput, the charge-coupled device (CCD) characteristics, the seeing condition, and the sky brightness.

In all the simulations in this paper, we take the major instrumental parameters from the official DES Exposure Time Calculator $^{37}$ (ETC) as listed in Table 1. The atmospheric throughput describes the fraction of light that passes through the atmosphere at zenith. The telescope throughput describes the fraction of light that passes through the telescope and arrives

\footnotetext{
36 The measured pixel scale on the DES SV data is closer to

0.263 arcsec pixel ${ }^{-1}$. Changing the pixel scale by this amount $(2.7 \%)$ would however not result in the significant difference in our analysis.

37 http://www.ctio.noao.edu/noao/content/Exposure-Time-Calculator-ETC-0
} 

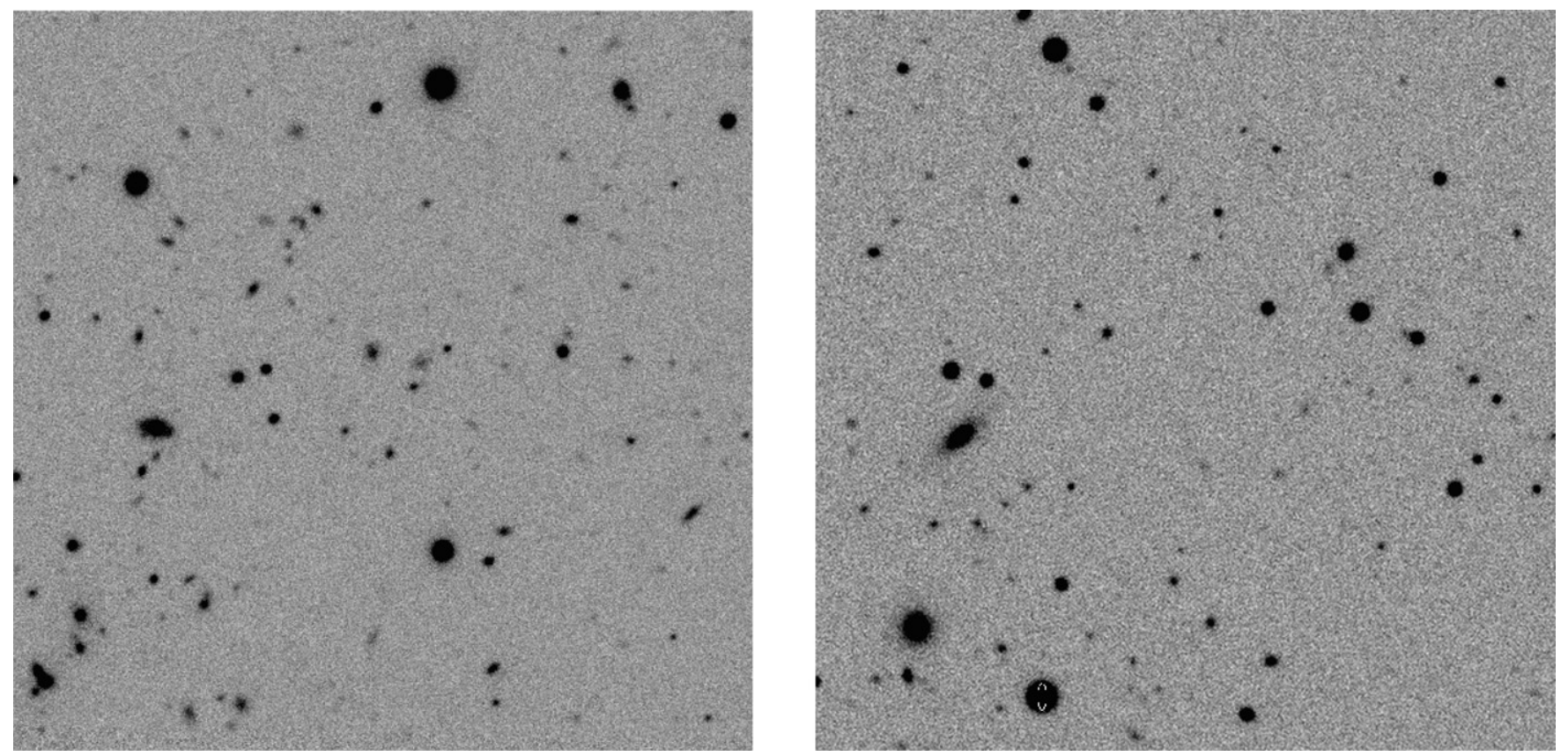

Figure 2. A $500 \times 500$ pixel region of an arbitrary $i$-band DES image (left) and its simulation counterpart (right). The scales in both images are the same. Note that the objects are not matched one-to-one in these images, but the statistical clustering and object properties appear qualitatively similar. Note also that the texture of the background is slightly different in the simulations compared to the data, indicating that improvements are needed for the background model.

Table 1

Basic Instrumental Parameters for the UFIG Image Simulations

\begin{tabular}{lccccc}
\hline \hline Filter & $g$ & $r$ & $i$ & $z$ & $Y$ \\
\hline Atmosphere throughput & 0.8 & 0.9 & 0.9 & 0.9 & 0.95 \\
Telescope throughput & 0.43 & 0.51 & 0.56 & 0.56 & 0.19 \\
Mean wavelength (nm) & 473 & 638 & 775 & 922 & 995 \\
Bandwidth (nm) & 147 & 141 & 147 & 147 & 50 \\
Quantum efficiency & 0.7 & 0.75 & 0.85 & 0.8 & 0.3 \\
\hline
\end{tabular}

at the focal plane. The mean wavelength and the bandwidth specify the basic properties of the filters. The quantum efficiency measures the fraction of photons that is converted into digital signal in the CCD. All quantities in this table are average values. Note also that we follow the DESDM convention and normalize the coadd images to either 90 ( griz-band) or 45 ( $Y$-band) second equivalent exposures.

On the other hand, the image-specific parameters (e.g., exposure time, seeing, background noise) are tuned to the specific data we wish to model. We use a circular Moffat PSF model with $\beta=3.5$ (Moffat 1969), which is typically a good description for ground-based optical PSFs. The PSF is assumed to be spatially constant in each image and have an FWHM (which can be specified for a Moffat profile with given $\beta$ parameter) equal to the mean seeing in the data of interest. Similarly, the background level is set so that the expected limiting magnitude agrees with the data (see the Appendix for details on the derivation of the background noise).

Figure 2 shows one arbitrary DES image in $i$ band and its simulation counterpart. Note that the objects in the images are not matched one-to-one, but the statistical clustering and noise properties appear qualitatively similar from visual inspection. We also note that due to the simplification in the background model (Gaussian noise plus Lanczos resampling), the texture of the background appears to be qualitatively different from the data.

\subsubsection{UFIG Image $\rightarrow$ DESDM Catalog}

In this step we run the DESDM software on the UFIG images to produce SExTRACTOR catalogs. First, the PSF model is estimated by PSFEx on each of the single-band coadd images. Then we follow the procedure implemented in DESDM and make a deep "detection image" by stacking the coadd images in three bands (riz). Objects are detected on the "detection image" but the properties of each object are measured on the single-band images using SExTRACTOR. The software versions used in this work are: SExTRACTOR v2.18.10, PSFEx v3.17.0 and SWARP v2.36.2. The configuration files for SEXTRACTOR and PSFEx can be found at: http://www.phys.ethz.ch/ ast/cosmo/bcc_ufig_public/bcc_ ufig_config.tar.gz

This is the most time-consuming step in the framework, as SEXTRACTOR carries out a large number of measurements and galaxy profile-fitting operations. However, depending on the specific science interest, it is possible to eliminate some of the SExtractor functionalities and make this step faster. For instance, eliminating the process of fitting galaxy profiles speeds up the procedure by a factor of $\sim 100$.

\subsubsection{DESDM Catalog $\rightarrow$ BCC Catalog}

Finally, to close the loop, the catalogs generated from SExTRACTOR above are matched to the input BCC catalogs by the position on the sky, and a matching file containing the galaxy ID's in the input and output catalog is written out. The matching process is sped up by first dividing each image into 20 smaller areas, and then matching within the subareas. It is this matching that gives us a model of the transfer function for DES data. We now have a mapping between the input signal from the sky and the final catalogs one uses for science.

\subsection{Data Volume and Computational Cost}

The images and catalogs in this work are generated on the Brutus cluster at ETH Zurich. The typical run time to generate 
Table 2

Summary for the Average Runtime on One Core and Size of Output Files for the SVA1 Simulations in This Work

\begin{tabular}{llcc}
\hline \hline Output & Run time & Format & Size \\
\hline Coadd image & $7.0 \mathrm{minutes}$ & FITS & $356 \mathrm{M}$ \\
SExTRACTOR catalog & $2.5 \mathrm{hr}$ & FITS & $53 \mathrm{M}$ \\
Matching file & 3.8 minutes & ASCII & $1.4 \mathrm{M}$ \\
\hline
\end{tabular}

Note. All numbers are quoted for one coadd image in one filter, and all data size are quoted after gzip compression.

the FITS image and SEXTRACTOR catalog for a $0.75 \times 0.75 \mathrm{deg}^{2}$ patch of sky in one filter band for our SVA1 simulation set (see Section 4) is summarized in Table 2, together with the file sizes. The run times are calculated for running with one core on AMD Opteron 6174/8380/8384 machines. Generally, the run time of the image generation scales with the number of photons, or exposure time and the run time for the analysis processes scale with the number of objects detected. The run time is dominated by the Source Extractor analysis process.

Note that Table 2 does not include the generation of the BCC catalogs upstream to this work, which includes the $\mathrm{N}$ body simulations and the input galaxy/star/quasar catalogs. To estimate the computational cost for the full end-to-end framework, one would also need to take into account these factors, which adds a total of $\sim 340 \mathrm{k} \mathrm{CPU}$ hours to the computational time.

\section{QUALITY ASSURANCE: FORWARD-MODELING THE DES SVA1 DATA}

In this section we present several basic quality assurance tests on the output catalog of the above simulation framework. The main goal is to show that our framework produces reliable catalogs that can be used for interpreting scientific data under well understood assumptions. For regimes where the simulations do not properly model the data, we identify areas for improvement in our model.

We set our target to model the DES SVA1 data set described in Section 2. We generate coadd images and catalogs covering the SVA1 footprint (Figure 1) in all 5 filter bands. In addition to the basic parameters listed in Table 1, we also use compiled maps for mean observational parameters from the data themselves (seeing, limiting magnitude, magnitude zeropoint). These maps are generated similar to the systematics maps described in Leistedt et al. (2013). For each of our images in each filter band, we find the corresponding region of sky in the maps. Then, we take the median value of the maps to be the observational parameters for this image. Note that for modeling another data set, even with the same instrument, the results could differ significantly. A portion of the SVA1 simulation output and supporting documentation can be found at http://www.phys.ethz.ch/ ast/cosmo/bcc_ufig_public/. The total number of coadd images is 480 in the griz bands and 432 in the $Y$ band.

Below we focus on examining three basic measurements of the detected objects in the images-magnitude, size, and object number counts.

\subsection{Magnitude}

Photometry lies at the center of many science analyses. Yet, in typical astronomical data, magnitude measurements and the corresponding errors are often hard to predict from first principles due to the noisiness of the data, the nonlinear nature of the measurement procedure, and the coupling to the objects' size and profile. We examine here the relation between the input and different measured magnitudes. Then we compare the general behavior of the different magnitude measurements in the SVA1 data compared with that in our simulations. Similar analyses have been done in Sevilla et al. (2011) and Rossetto et al. (2011) for early DES simulations.

In Figure 3 we show the distribution of the difference between measured and input magnitude as a function of input magnitude for three different magnitude estimates from SEXTRACTOR (MAG_AUTO, MAG_MODEL and MAG_DETMODEL) on one arbitrarily selected $i$-band image. MAG_AUTO is measured by summing the flux in an ellipse scaled to the Kron radius (Kron 1980); MAG_MODEL is measured by fitting the object with a given model and estimating the flux for this model; MAG_DETMODEL is similar to MAG_MODEL but first carries out the model fitting on the detection image, and then fits the overall normalization of this model to each single-band image separately. MAG_DETMODEL thus has a consistent galaxy model for the same galaxy across all filters, which is primarily useful for color measurements. For SVA1, MAG_MODEL and MAG_DETMODEL use a single exponential profile for the galaxy model.

The general trend between all three estimates is that the measured magnitudes tend to be biased high and that faint objects have larger photometric errors than bright objects. The bias is due to the fact that the magnitudes are all calculated within some finite pixels defined by the signal-to-noise of each pixel, whereas in reality, light can fall much further out. For the stars, the bias is at the 0.01-0.02 level at the bright end, with MAG_AUTO slightly higher than the other two. This is sensible as the fitting methods (MAG_MODEL and MAG_DETMODEL) does account for some of the low-level wings. Model fitting also results in smaller scatter at the faint end and the sharp turnoff at the very bright end, where the model fails to fit bright star profiles. For galaxies, there is a small "bump" feature at magnitude $\sim 20$. The feature is a result of the input galaxy model, where galaxies have different distribution of profiles above and below $i=20$ (Equation (1)). The galaxy MAG_AUTO measurements behave similarly to those for the stars with slightly more scatter. MAG_MODEL and MAG_DETMODEL, however, do not improve significantly the magnitude measurements compared to MAG_AUTO. This could indicate that the model for the galaxy profiles used by MAG_MODEL and MAG_DETMODEL is insufficient for the wide range of galaxy profiles in the simulations (and in data). We also see that MAG_MODEL is less biased compared to MAG_DETMODEL. This is because MAG_DETMODEL derives the galaxy model from the detection image ( $r i z$-coadd) instead of the image where the magnitude is measured. Note that the difference would be larger in real data, where unlike in our simulations, the galaxy and the PSF profiles change in different filter bands.

In Figure 4 we show the magnitude error against magnitude for one arbitrary $i$-band DES image and the corresponding UFIG simulation. We examine the behavior of three different magnitude estimates in the SExTRACTOR catalog. All objects in both catalogs are plotted. The broad features in the different panels agree between the simulation and the data with some discrepancies that are expected from the simplifications and assumptions described in Section 3. First, in the MAGERR_AUTO-MAG_AUTO panel the data and simulations agree down to $i \sim 24.5$, but there are more objects in the simulations compared to the data at $i>24.5$. This shows that the simulation is able to reproduce the behavior of the magnitude error at $i<24.5$, which is sufficiently 

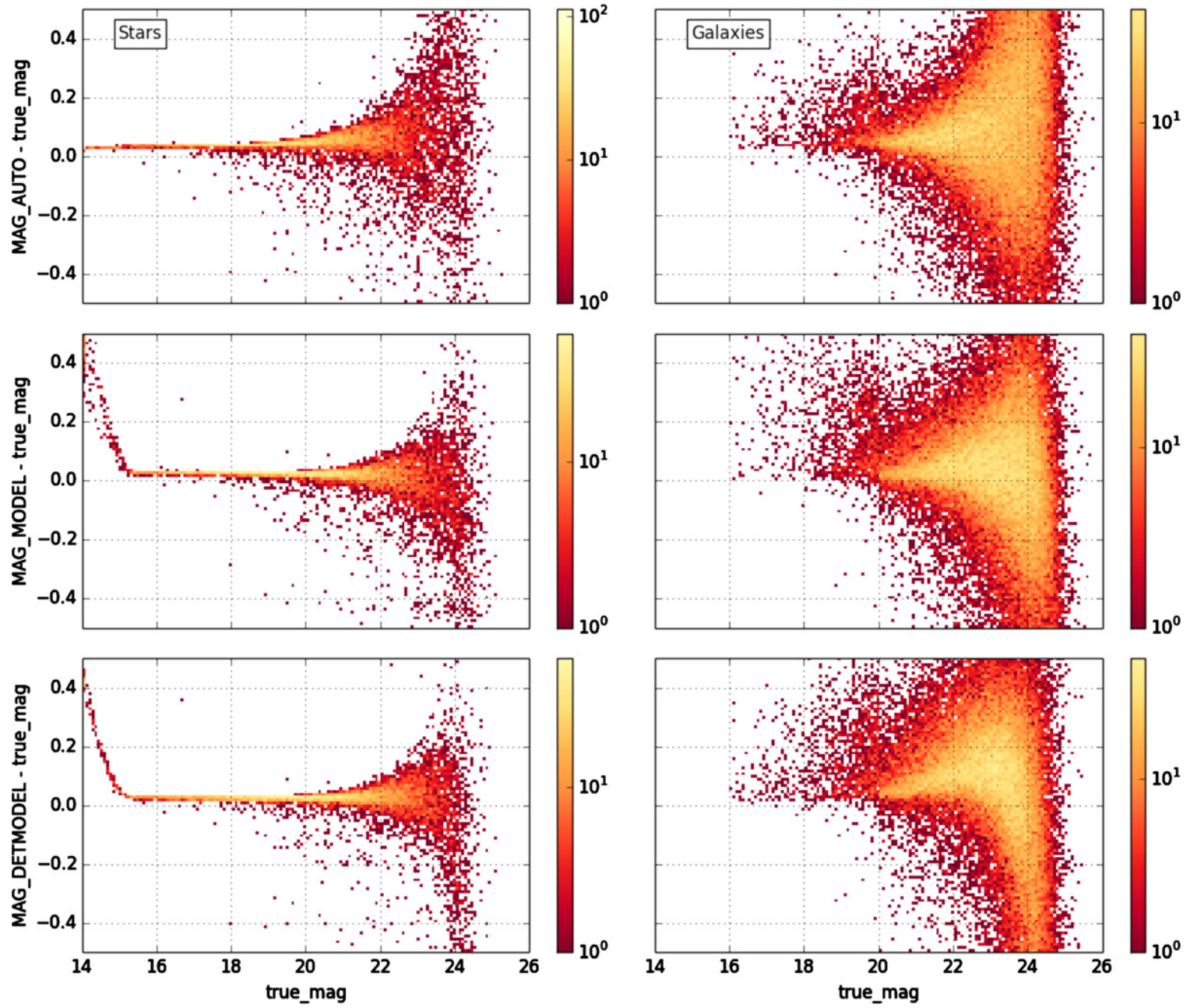

Figure 3. Distribution of the differences in three magnitude measurements and the true input magnitude as a function of the input magnitude. From top to bottom are the SEXTRACTOR magnitudes MAG_AUTO, MAG_MODEL, and MAG_DETMODEL. Left and right panels are for stars and galaxies respectively. All plots are generated for one arbitrary $i$-band image in our simulation. Note that the color scales are logarithmic.
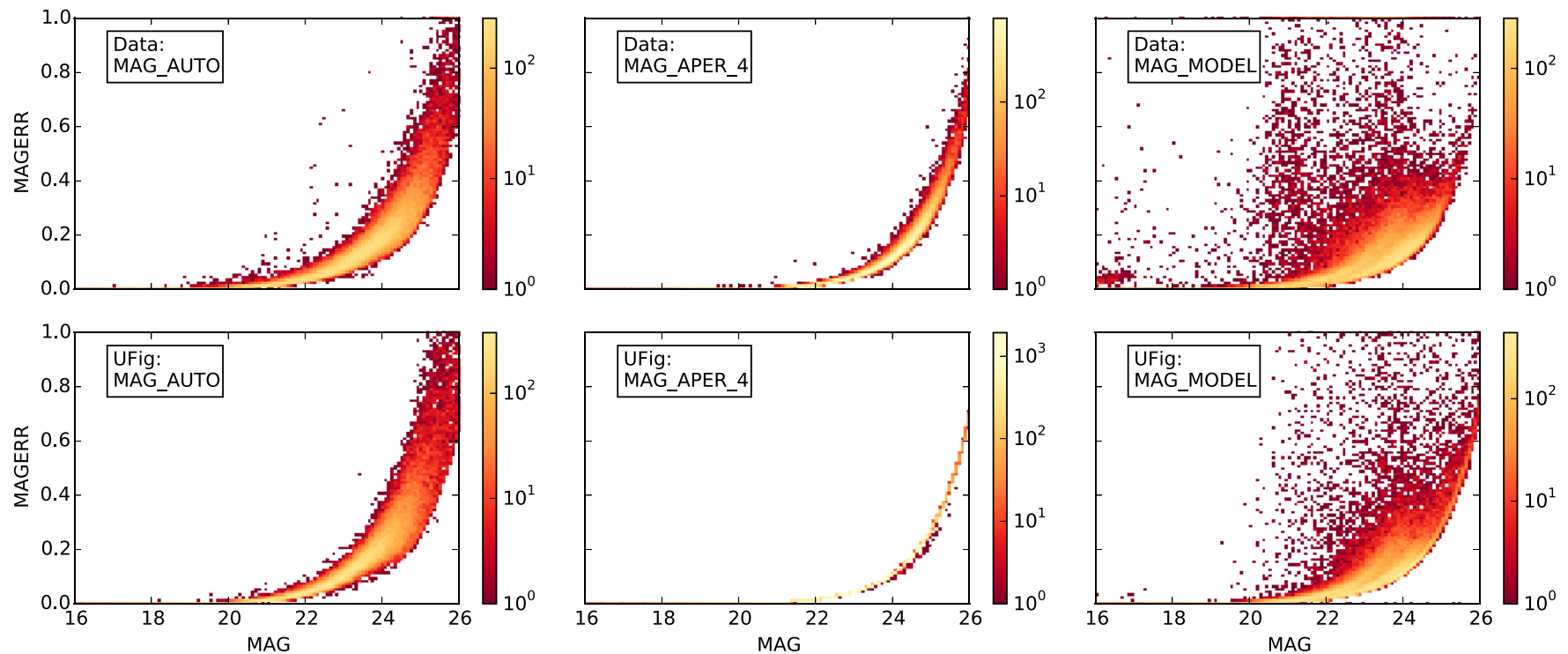

Figure 4. Distribution of three magnitude measurements and the associated errors as quoted from the SEXTRACTOR output. From left to right are the SExTRACTOR magnitudes MAG_AUTO, MAG_APER_4 (2 arcsec), and MAG_MODEL. The top row shows that measured from one arbitrary $i$-band SV image and the bottom shows the measurement from the corresponding simulated image. The color scales are logarithmic. Note that the middle bottom panel shows that most of the data points lie on a very tight line in this parameter space. 

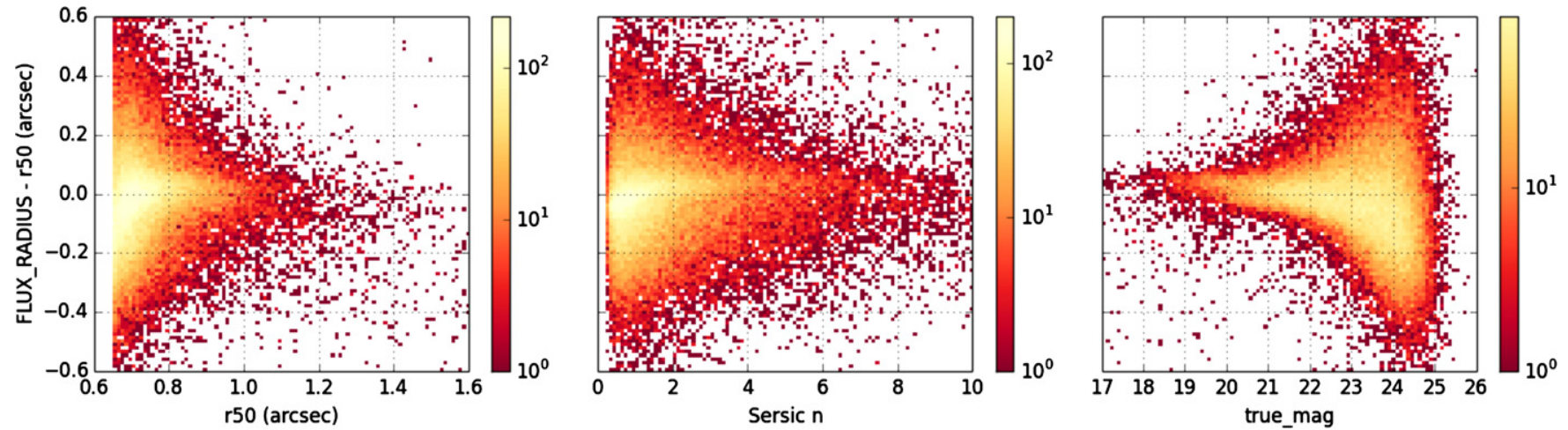

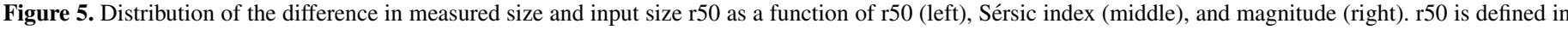
Equation (2). All plots are generated for one arbitrary $i$-band image in our simulation. And more that the color scales are logarithmic.

deep for DES. For the fainter objects, one should take caution when interpreting results from the simulations in this regime. Second, the MAGERR_APER_4-MAG_APER_4 relation in the simulation lies on top of that from data. This confirms that our noise model behaves as expected (see the Appendix). The data contains more scatter compared to the simulations. This is expected as the limiting magnitude varies within an image in data, while we have assumed it to be constant in our simulations. Finally, for the MAG_MODEL-MAGERR_MODEL panel, both data and simulation show an overall more complicated shape of the distribution. The same qualitative feature can be seen in both plots, such as the sharp drop of numbers at MAGERR_MODEL $\sim 0.2$, the faint could of objects with large MAGERR_MODEL at MAG_MODEL $~ 24$. These indicate that our model of the intrinsic galaxy morphology (size and Sérsic index) is reasonable. The details in the two distributions are however different. This is an indication that improvements are needed in the future in this area, and one should use caution when using MAG_MODEL in our simulations.

\subsection{Size}

The first-order morphological information we can measure from an object's image is its observed size. The measured size of an object in a noisy image is usually defined in terms of the flux in a set of pixels that are assigned to this object-for example, the parameter FLUX_RADIUS in SEXTRACTOR refers to the radius within which $50 \%$ of the total flux is enclosed. The measured size is thus coupled with magnitude measurements and is sensitive to the noise in the image, the PSF, and the intrinsic object profile.

In Figure 5, we show the distribution of the difference between measured object size and input size (r50) as a function of input size, Sérsic index and true magnitude for all detected objects in one arbitrary $i$-band image. The "input size" r50 here refers to the expected half-light radius of the object after convolving with the PSF. We calculate it via the following empirical relation:

$$
\mathrm{r} 50=\sqrt{\mathrm{r} 50_{\text {in }}^{2}+\mathrm{rSF}^{2} / 2.355},
$$

where $\mathrm{r} 0_{\text {in }}$ is the intrinsic half-light radius given by the $\mathrm{BCC}$ catalog and $\mathrm{r}_{\mathrm{PSF}}$ is the seeing for that image. The numerical factor 2.355 is derived empirically to account for the change of the apparent galaxy size when convolved with the PSF. Note that Equation (2) is only an approximate relation between $\mathrm{r}^{5} 0_{\text {in }}$ and r50. Nevertheless, we use it here to illustrate the qualitative behavior of the size measurements in our catalogs.

Figure 5 shows that small, faint, disk-like galaxies have larger errors on the size measurement. The distribution of the errors are

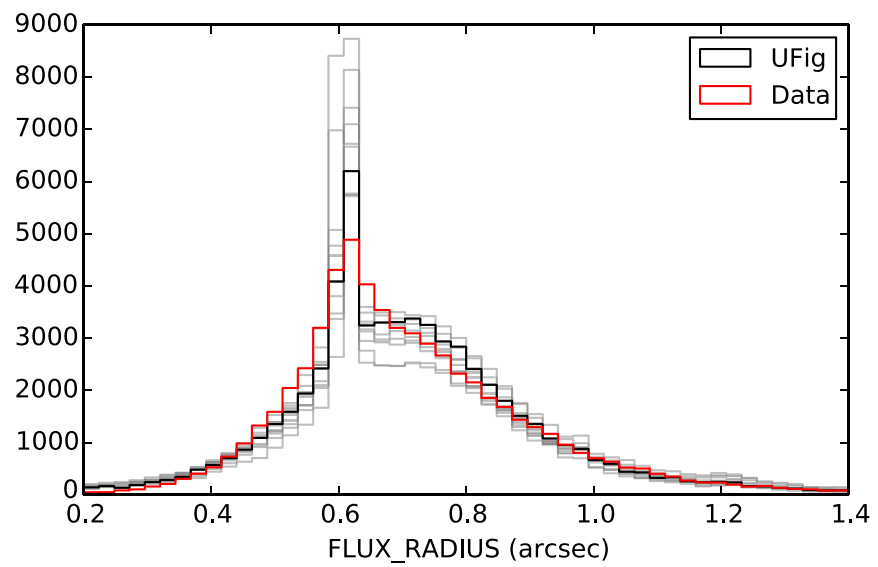

Figure 6. Measured size distribution for all objects from the UFIG simulations (black) compared to the SVA1 data (red) in the same area. The gray lines show the same distribution as the black line, but for other tiles in our SVA1 simulation that have limiting magnitudes and seeing conditions within $1 \%$ of the region of interest. The disagreement in the distributions is consistent with the variation from cosmic variance.

asymmetric with more objects biased small. The origin of the asymmetry comes from the fact that SEXTRACTOR measures the sizes with a finite set of pixels while the galaxy profile generally extends beyond that.

In Figure 6 we compare the measured size distribution of all the detected objects in one arbitrary $i$-band image in the SVA1 data and the corresponding simulation. Also overlaid in gray are 10 other size distributions from the simulations that have limiting magnitude and seeing values within $1 \%$ of this image, these curves give an estimate of the variation in the size distribution due to cosmic variance. We find that the measured size distribution in our simulations are consistent with that measured in data within cosmic variance. The narrow peak at FLUX_RADIUS $\sim 0.6$ arcsec corresponds to the seeing value for this image. The peak is broadened in the data since unlike in the simulations, there exists seeing variation within each image. The size distribution of the remaining objects (mostly galaxies) match very well between the data and simulations, especially on the high and low end where it is less sensitive to our assumption of constant seeing. Seeing variation is thus one important factor to improve in future developments.

\subsection{Number Density}

Finally, we examine the detected star and galaxy number densities. This is important because it simultaneously checks the 

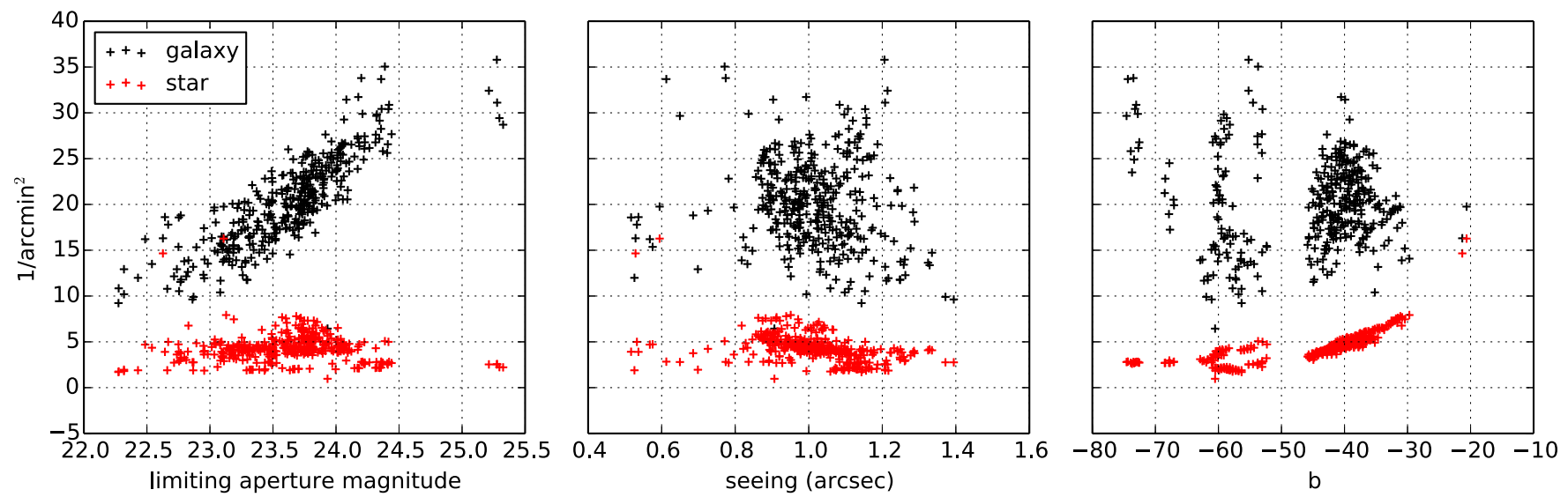

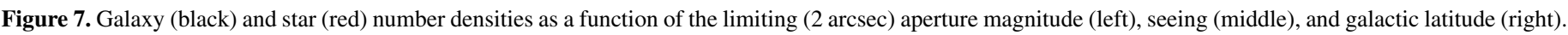

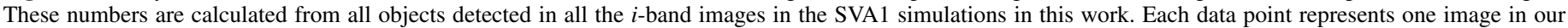
simulation. The discontinuous distribution of data points in the $x$ axis of the right panel reflects the SVA1 footprint.

Table 3

Object Number Density (Per Sq. Arcmin) from Data and Our Simulations under Different Magnitude (MAG_AUTO) Cuts

\begin{tabular}{lrr}
\hline \hline & Data & Simulation \\
\hline All objects & 27.79 & 31.05 \\
$15<i<19$ & 1.06 & 1.01 \\
$15<i<21$ & 3.43 & 3.85 \\
$15<i<23$ & 11.95 & 12.82 \\
\hline
\end{tabular}

input source distribution, the image simulation, and the analysis software.

In Figure 7 we show the star and galaxy number density in all the $i$-band-simulated SVA1 images as a function of limiting magnitude, seeing, and galactic latitude. We observe that the general behavior of the number counts follows expectation. In deeper fields the number density of stars and galaxies both increase. The group of data points on the far right are the SN fields (see Figure 1) where the total exposure time is significantly longer than in the rest of the fields. Note, however, that the input BCC catalogs are not necessarily complete at those magnitudes, thus one should be careful in interpreting the results there and only treat those data points as lower bounds. The dependence on seeing is also expected (keeping in mind that seeing and limiting magnitude are not independent)_higher seeing gives slightly lower number density since the signal-to-noise of the objects decreases going to higher seeing. Finally, we look at the correlation between number density and the galactic latitude as a check for the input source catalog. We find that the stellar density, as expected, increases toward the galactic plane, whereas the galaxies do not. The discontinuous distribution of data points in the $\mathrm{x}$ axis reflects the SVA1 footprint.

To compare the number counts derived from simulations and data, we calculate the mean source density as a function of magnitude cuts for both the SVA1 catalog and our simulations. We use all objects in the catalogs and do not make distinction between stars and galaxies. We choose to do so to avoid making choices in the object selection. This also means that we are accounting for spurious detections from noise, blended objects, and artifacts. Table 3 summarizes our results. We find that the data and the simulations agree at the $\sim 10 \%$ level. The agreement is best at the bright end, where the errors in the object property as well as the noise is more accurate. The agreement is not perfect, but rather encouraging, given the current uncertainty in the source catalog, the galaxy profile model and the noise model.

\section{APPLICATIONS}

In this section we describe two example cases where we use the simulation products described in Section 4 to help answer questions in the data analysis process. The advantage of using this framework is that the simulations are sufficiently realistic, yet we have full control over every stage of the simulation and data processing pipeline. For the use of our simulations in scientific analyses on the DES SV data, see E. Rykoff et al. (in preparation).

\subsection{Star-Galaxy Classification}

Identifying stars and galaxies in optical images is one of the most basic operations in the data analysis pipeline. Depending on the science application, one would demand good efficiency and/or purity in the star sample and/or the galaxy sample. For example, in weak gravitational lensing, one would require a pure star sample for the PSF estimation, and a pure galaxy sample for uncontaminated lensing signal. On the other hand, for the study of galaxy evolution, the completeness of the galaxy sample is also important in order for one to extract global behaviors of the galaxy population. We define the star/galaxy classification efficiency $(E)$ and purity $(P)$ below:

$$
\begin{gathered}
E(\mathrm{X})=\frac{\text { Number of objects correctly identified as } \mathrm{X}}{\text { Number of all } \mathrm{X}} \\
P(\mathrm{X})=\frac{\text { Number of objects correctly identified as } \mathrm{X}}{\text { Number of objects identified as } \mathrm{X}},
\end{gathered}
$$

where $\mathrm{X}$ is either stars or galaxies.

The problem is challenging, however, in typical groundbased imaging data. With typical seeing and noise conditions in these images, small, faint galaxies become indistinguishable from stars. A wide range of techniques has been developed to resolve this problem (Henrion et al. 2011; Fadely et al. 2012; Soumagnac et al. 2013). Standard star-galaxy classifiers use morphological information of the stars, more advanced ones incorporate also the color information (Pollo et al. 2010). The simulations from this work, with both realistic image characteristics and color information, offer a generic tool for different methods to be tested on before applying to data. 

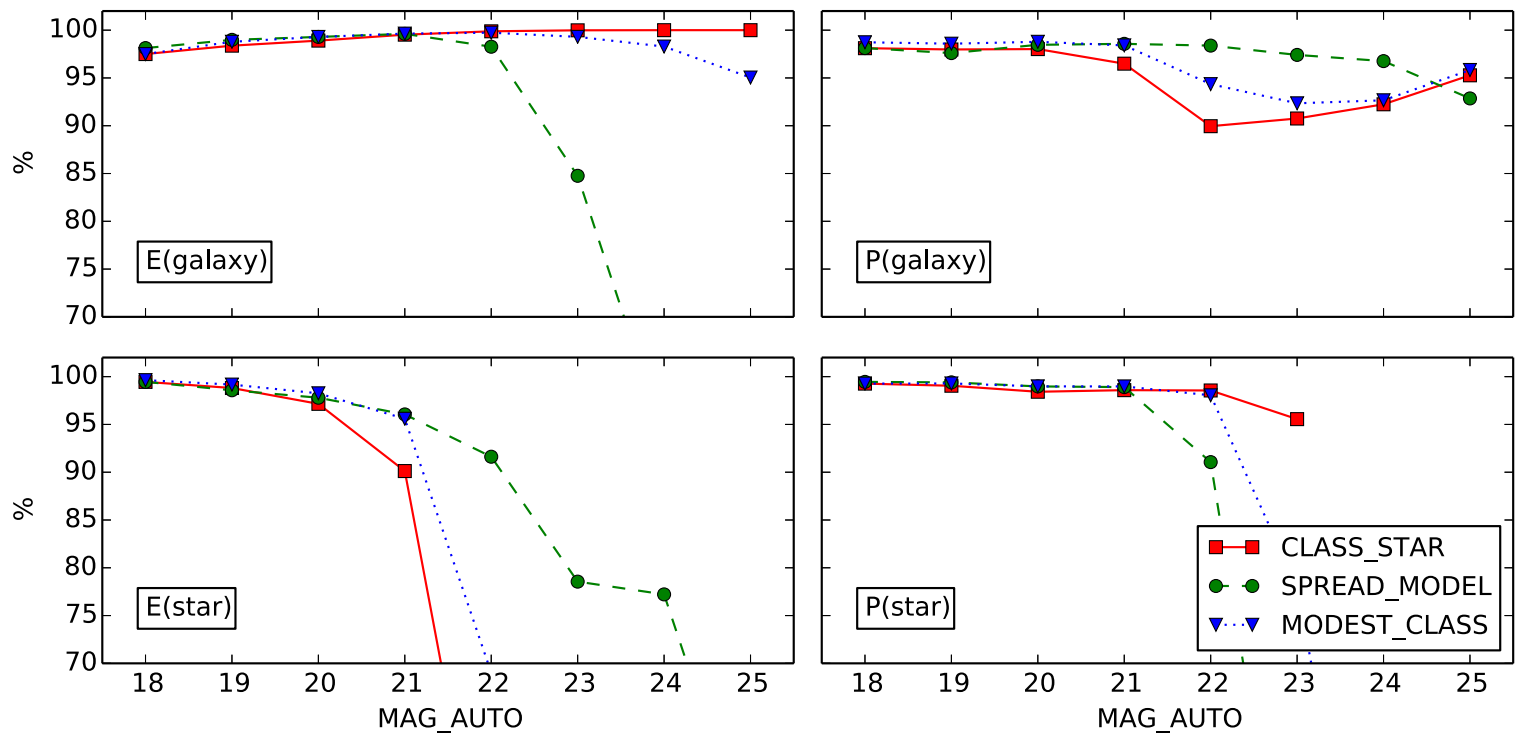

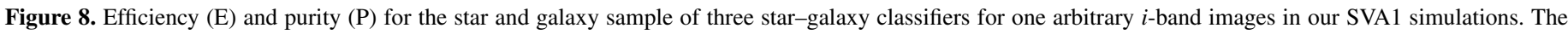
three classifiers are described in Table 4.

Table 4

Cuts Used in the Three Classifiers: CLASS_STAR, SPREAD_MODEL and MODEST_CLASS

\begin{tabular}{lc}
\hline \hline Galaxies & Stars \\
\hline CLASS_STAR $<0.95$ & CLASS_STAR $>0.95$ \\
SPREAD_MODEL $>0.002$ & SPREAD_MODEL $<0.002$ \\
MODEST_CLASS $=1^{\mathrm{a}}$ & MODEST_CLASS $=2^{\mathrm{b}}$ \\
\hline
\end{tabular}

Notes. For a full description of MODEST_CLASS, see footnote below. All of these cuts have an additional cut on FLAGS $<=3 \sigma$ and $5 \sigma$ detection.

a MODEST_CLASS $=1$ : (FLAGS $<=3)$ AND(NOT (CLASS_STAR > 0.3) AND (MAG_AUTO $<18.0)$ OR ((SPREAD_MODEL+ $3 *$ SPREADERR_MODEL) $<0.003)$ OR ((MAG_PSF $>30.0)$ AND (MAG_AUTO < 21.0))).

b MODEST_CLASS $=2:($ FLAGS $<=3)$ AND ((CLASS_STAR > $0.3)$ AND (MAG_AUTO $<18.0)$ AND (MAG_PSF $<30.0)$ OR (((SPREAD_MODEL+ $3 *$ SPREADERR_MODEL) $<0.003)$ AND $(($ SPREAD_MODEL+3*SPREADERR_MODEL $)>-0.003)))$.

Moreover, since the simulations are tailored for a specific set of data, one can consistently evaluate the effect of star-galaxy separation on specific science measurements performed on the same data set.

Here, we show an example of quantifying the performance of three single-band cut-based star-galaxy classifiers, which are based solely on the SExtractor catalogs. The three classifiers which we label as CLASS_STAR, SPREAD_MODEL, and MODEST_CLASS are described in Table 4. CLASS_STAR is a pretrained Artificial Neural Network method that uses several of the photometric and shape information in the SEXTRACTOR catalogs. It works well at the bright end but is limited by requiring the user to know the approximate seeing of the image prior to processing. SPREAD_MODEL (Mohr et al. 2012; Bouy et al. 2013) uses pixel-level morphological information and compares the profile of each object with the local PSF. For faint objects, where the classification is most challenging, CLASS_STAR with the current settings tends to classify all objects as galaxies at the faint end while a naive SPREAD_MODEL classifier with constant threshold tends to classify all objects as stars. MODEST_CLASS is a new classifier used for SVA1 Gold that has been developed empirically and tested on DES imaging of COSMOS fields with Hubble Space Telescope ACS imaging. It is primarily based on SPREAD_MODEL, and attempts to fix the faint galaxy classification by including the error on SPREAD_MODEL.

We evaluate the $E$ and $P$ statistics for stars and galaxies on one arbitrary $i$-band image in our SVA1 simulations as a function of the measured MAG_AUTO. The results are shown in Figure 8. In this particular image, the simulations confirm nicely what we expect from the construction of the three classifiers (see above). For example, for galaxies, SPREAD_MODEL gives high $\mathrm{P}$ and low E at the faint end, CLASS_STAR behaves in the opposite direction, and MODEST_CLASS sits between the two. We also see that all classifiers perform well at the bright end while degrading at the faint end.

In Figure 9, we plot the median of the E and P statistics for galaxies and for all the SVA1 simulations as a function of seeing. The statistics is evaluated at $18.5<$ MAG_AUTO $<19.5$ and $22.5<$ MAG_AUTO $<23.5$ to illustrate the global performance of the different classifiers at bright and faint magnitudes. We find that CLASS_STAR is unstable at the bright end $i \sim 19$, while the other two perform well. At the faint end, MODEST_CLASS improves from SPREAD_MODEL in E(galaxy), consistent with Figure 8. There is mild dependence on seeing for SPREAD_MODEL and MODEST_CLASS at the bright end and all classifiers at the faint end. Interestingly, the galaxy classification purity rises going toward larger seeing and drops after $\sim 1.05$ arcsec.

As there are simplifications in both our galaxy and PSF, we do not expect these results should reproduce quantitatively exactly the same in data. However, the simulations allow us to study the response of different star-galaxy classifiers to observational parameters and object properties. Understanding the physical interpretation for their behaviors in the simulations then helps us quantify the contamination in our star/galaxy sample in data.

\subsection{Proximity Effects on Object Detection}

Object detection software for imaging data, such as SExTRACTOR, relies on identifying a group of pixels that have values above the local background level at some predefined signalto-noise threshold. As a result, the probability of detecting an 

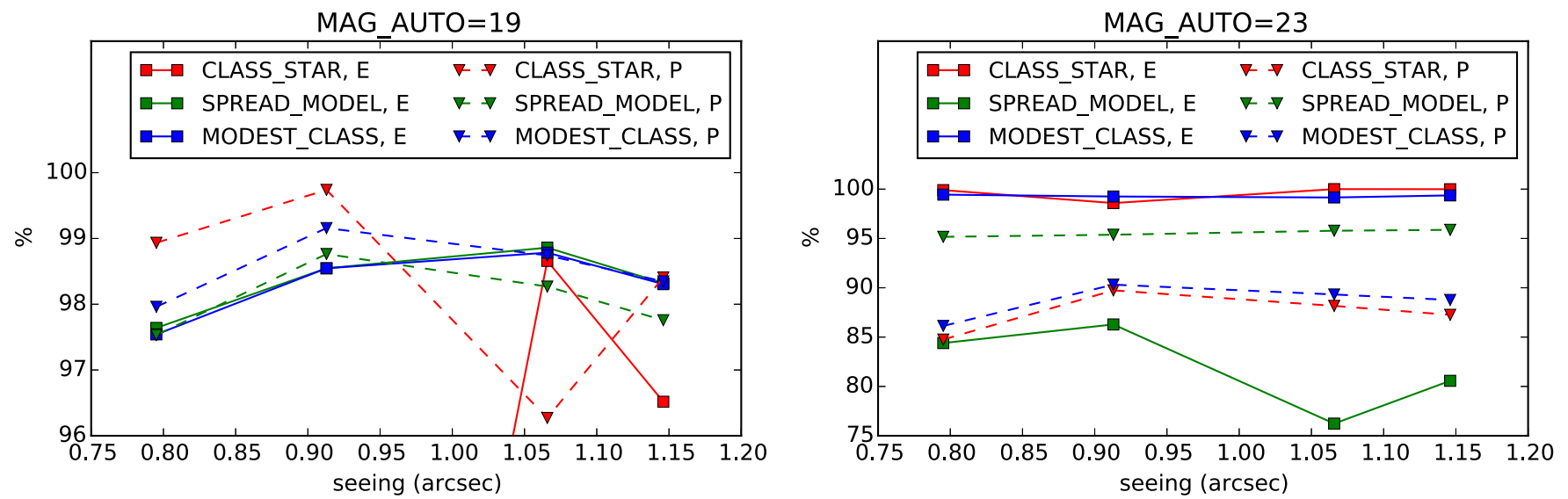

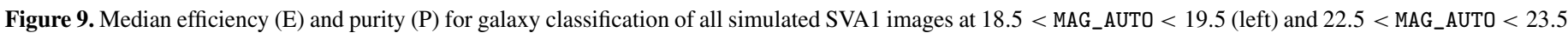
(right), as a function of seeing of that image. The three classifiers are described in Table 4. Note that the $y$ axis has very different scales.

object depends on the object brightness and the local pixel values around that object-these pixels contain not only the sky background but also photons from other objects nearby. The proximity effect on object detection refers to the fact that for the same object and sky background, we are less likely to detect it when nearby bright objects exist. This effect is especially pronounced in crowded environments such as galaxy clusters or dense stellar fields (Melchior et al. 2014; Zhang et al. 2014), but can also affect more generally the clustering statistics for large-scale structure (Ross et al. 2012; Huff \& Graves 2014).

Calibrating the effect from data itself is possible, but can be coupled with other factors such as photometric errors and star-galaxy classification. On the other hand, simple cataloglevel simulations are inefficient for this specific problem, as the object detection algorithm is a highly nonlinear operation and needs to be performed on images. Image-level simulations, such as that developed in this work are ideal for this test, as it contains the following key features that are required to perform this analysis: (1) realistic spatial distribution (clustering) of galaxies and stars, (2) realistic observed magnitude distribution of stars/galaxies and morphology distribution for galaxies, and (3) image-level simulations that are processed through the same object detection software as the data. In this section, we demonstrate an example where we quantify via simulations the degradation in detection efficiency due to the proximity effect. The approach of using simulations to correct for these effects has been used in recent literature. For example, Melchior et al. (2014) used simulations from the Balrog ${ }^{38}$ code to assess how the crowded cluster environment reduces the probability of performing weak lensing measurements near the center of galaxy clusters.

We calculate the detection efficiency $F_{\text {det }}(r)$ at a distance $r$ around a particular sample of objects (e.g., bright galaxies). $F_{\text {det }}(r)$ is defined as

$$
F_{\text {det }}(r)=\frac{\sum_{i}^{n} N_{i, \text { det }}(r)}{\sum_{i}^{n} N_{i, \text { true }}(r)},
$$

where $i$ is summed over the $n$ objects in this sample of interest, $N_{i, \text { det }}(r)$ is the number of objects detected at a distance $r$, and $N_{i, \text { true }}(r)$ is the true number of objects at this distance. Without the proximity effect, we expect the $F_{\text {det }}(r)$ curve to be flat.

In Figure 10 we show the $F_{\text {det }}(r)$ for an arbitrary $i$-band image in our SVA1 simulations. Here we set up the calculation to

\footnotetext{
38 https://github.com/emhuff/Balrog
}

estimate the detection efficiency of galaxies at $18<i<24$ in the surrounding of other galaxies in different (true) magnitude bins. For clarity, we will refer to the objects responsible for the drop in detection efficiency the "center" objects and the objects being detected the "source" objects. We would like to know how many source galaxies are missing in the magnitude range of $18<i<24$ because there is a center galaxy nearby. We find that the proximity effect is most severe in the surrounding of bright center galaxies, and the effect is seen up to several arc seconds away from the center galaxy. In the most severe case in this test $(18<i<19$ center galaxies), the detection of the source galaxies is $50 \%$ less efficient at $\sim 4$ arcsec. For comparison, the average measured galaxy size (FLUX_RADIUS) in this image is $\sim 0.96$ arcsec.

On the right panel of Figure 10 we only show the detection efficiency for the magnitude bin $19<i<20$, and overlay gray curves calculated from 10 random fields that have a range of limiting magnitude and seeing conditions. The gray curves agree well with the blue curves within error bars. This shows that neither cosmic variance nor seeing and limiting magnitude play a significant role in this calculation, i.e., the proximity effect is roughly at the same level for all galaxies in this magnitude bin across the sky under any observational conditions. However, if we calculate the same effect around stars in the same magnitude bin, as shown by the black curve, the shape of the curve changes and the detection efficiency increases at small separations. This is as expected since the stars have less extended profiles and are less likely to affect measurements in its surrounding pixels.

One can imagine many more similar tests using these simulations to quantify the proximity effects as a function of crowding, galaxy size and profiles etc., which would be required depending on the science analysis of interest. We will not carry out the analyses here, but only point out via the example above that by properly using simulations, one can correct for the proximity effects in the data that are otherwise difficult to estimate.

\section{CONCLUSIONS}

Precision cosmology in ongoing and future optical surveys critically depend on the control of systematic effects. In this generation, end-to-end simulations will play an important role in understanding these systematic effects. In this paper we describe a framework for forward-modeling the transfer function for the DES that takes the astronomical sources to realistic pixel-level data products such as images and catalogs. The same framework can be adjusted for other surveys and data sets. 

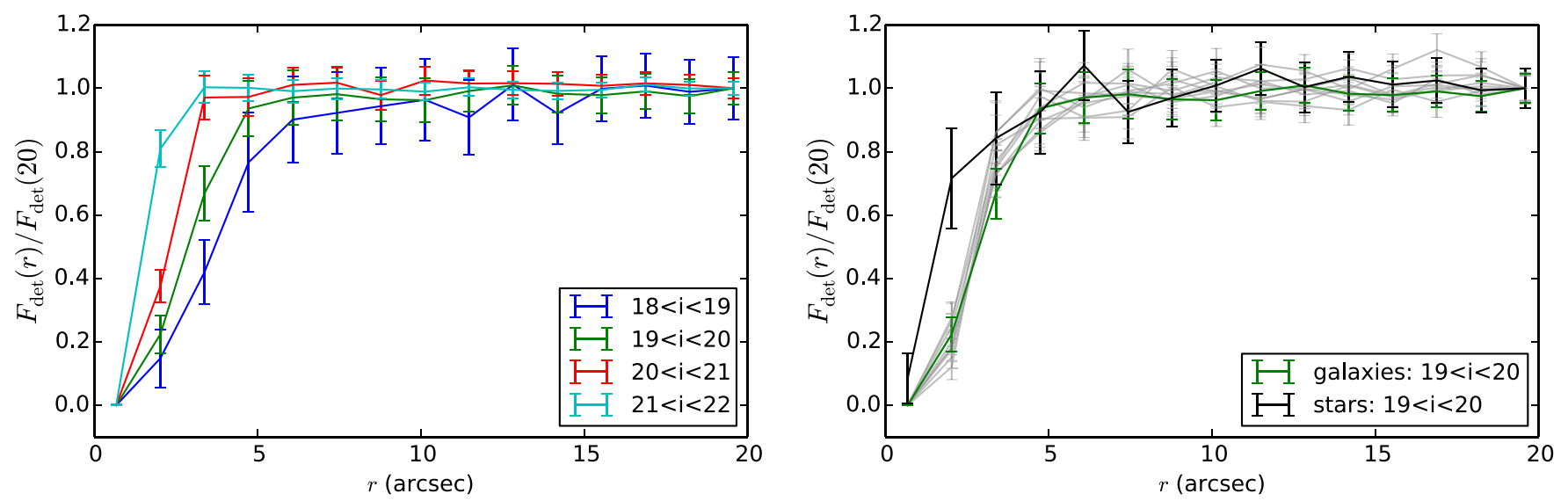

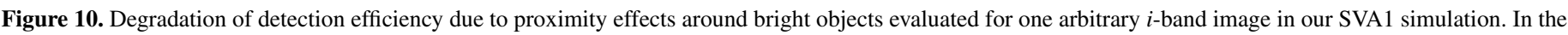

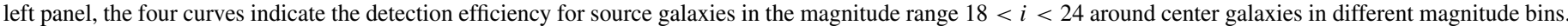

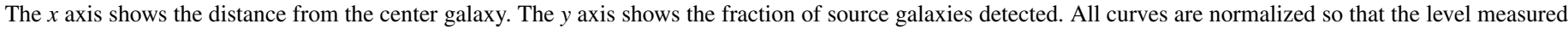

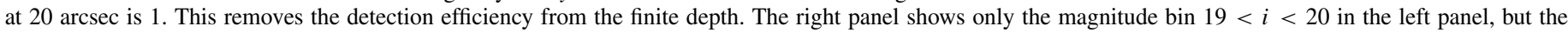

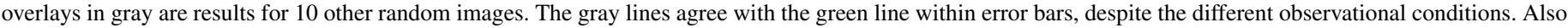
plotted in black is the result when we replace the center galaxies with stars, which results in a qualitatively different shape of curve.

We use the Blind Cosmology Challenge (BCC) catalogs as the source of astronomical objects, and simulate realistic images using the Ultra Fast Image Generator (UFIG). We then perform image analysis to output catalog-level products. We demonstrate the use of this framework by forward-modeling the early SV data products from DES. We design the simulations and the analysis procedure to mimic closely that of the SV data, and show that our simulations reproduce many major characteristics of the data. There are small differences between the data and the simulations in certain areas of parameter spaces (e.g., small faint objects), but they can be explained by our simplified models and do not affect significantly the usage of the simulation as long as one is aware of the simplifications. By connecting the output measurement back to the input object by object, we have a powerful tool to investigate data-related systematic issues. We present two examples of such usage looking at star-galaxy classification and proximity effects.

This is the first implementation of such end-to-end simulation efforts for ongoing large optical surveys. In the process we have made simplifications that we understand and will improve on continuing into future work. These include (1) more sophisticated models for the source morphological distribution, (2) more realistic and spatially varying models for the PSF and the background, and (3) extending the current framework to also model the single-exposure images and the coadd procedure. This constantly developing simulation framework that forwardmodels the data side-by-side as DES continues to release data provides a powerful tool to understand and interpret data in a clean and controlled fashion. The concept can also be extended to future surveys, where the need to understand details in the data products is even more demanding.

We are grateful for the extraordinary contributions of our CTIO colleagues and the DES Camera, Commissioning and Science Verification teams in achieving the excellent instrument and telescope conditions that have made this work possible. The success of this project also relies critically on the expertise and dedication of the DES Data Management organization.

We thank Gary Bernstein, Eric Huff, Tesla Jeltema, Huan Lin, and Felipe Menanteau for helpful comments and discussions on the paper. C.C., A.R., A.A., and C.B. are supported by the Swiss National Science Foundation grants 200021-149442 and 200021-143906. M.T.B., R.H.W., E.R., and M.R.B. acknowledge support from the Department of Energy contract to SLAC National Accelerator Laboratory No. DE-AC376SF00515. B.L. is supported by the Perren Fund and the IMPACT Fund. H.V.P. is supported by STFC and the European Research Council under the European Community's Seventh Framework Programme (FP7/2007- 2013)/ERC grant agreement No. 306478-CosmicDawn. A.C.R. is supported by the PROGRAMA DE APOIO AO POS-DOUTORADO NO ESTADO DO RIO DE JANEIRO - PAPDRJ. D.G. was supported by SFB-Transregio 33 "The Dark Universe" by the Deutsche Forschungsgemeinschaft (DFG) and the DFG cluster of excellence "Origin and Structure of the Universe." A.P. is supported by DOE grant DE-AC02-98CH10886. J.Z. acknowledges support from the European Research Council in the form of a Starting Grant with number 240672.

Funding for the DES Projects has been provided by the U.S. Department of Energy, the U.S. National Science Foundation, the Ministry of Science and Education of Spain, the Science and Technology Facilities Council of the United Kingdom, the Higher Education Funding Council for England, the National Center for Supercomputing Applications at the University of Illinois at Urbana-Champaign, the Kavli Institute of Cosmological Physics at the University of Chicago, Financiadora de Estudos e Projetos, Fundação Carlos Chagas Filho de Amparo à Pesquisa do Estado do Rio de Janeiro, Conselho Nacional de Desenvolvimento Científico e Tecnológico and the Ministério da Ciência e Tecnologia, the Deutsche Forschungsgemeinschaft and the Collaborating Institutions in the Dark Energy Survey.

The Collaborating Institutions are Argonne National Laboratory, the University of California at Santa Cruz, the University of Cambridge, Centro de Investigaciones Energeticas, Medioambientales y Tecnologicas-Madrid, the University of Chicago, University College London, the DES-Brazil Consortium, the Eidgenössische Technische Hochschule (ETH) Zürich, Fermi National Accelerator Laboratory, the University of Edinburgh, the University of Illinois at Urbana-Champaign, the Institut de Ciencies de l'Espai (IEEC/CSIC), the Institut de Fisica d'Altes Energies, Lawrence Berkeley National Laboratory, the LudwigMaximilians Universität and the associated Excellence Cluster Universe, the University of Michigan, the National Optical Astronomy Observatory, the University of Nottingham, The Ohio 

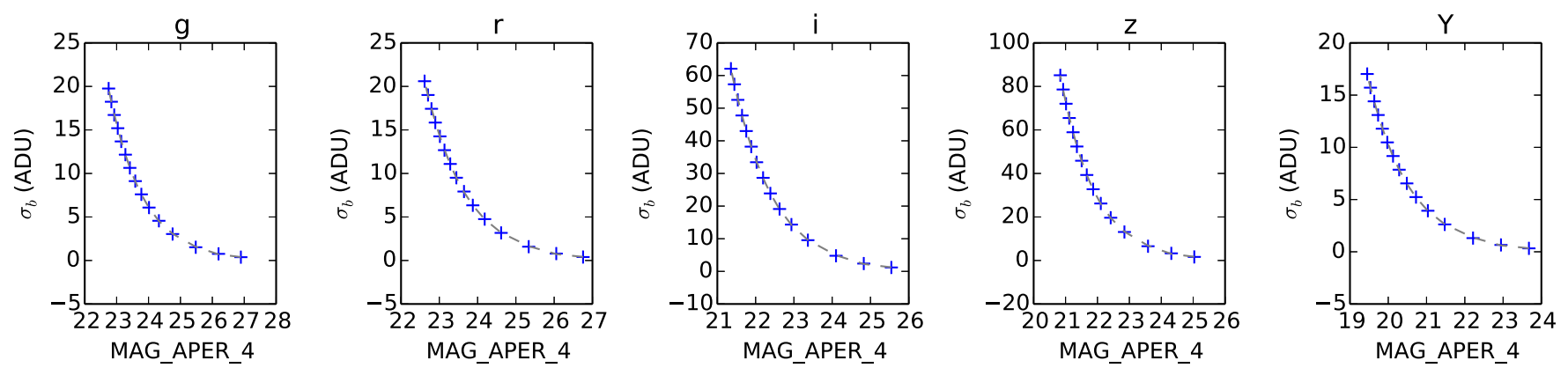

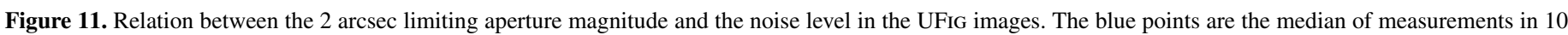
random fields and the gray dashed line is the fourth-order polynomial fit to these data points.

State University, the University of Pennsylvania, the University of Portsmouth, SLAC National Accelerator Laboratory, Stanford University, the University of Sussex, and Texas A\&M University.

This paper has gone through internal review by the DES collaboration.

\section{APPENDIX}

\section{NOISE LEVEL IN UFIG IMAGES}

The noise level in images affects object detection, photometry measurements, and the completeness of the final catalog. As a result, we want to simulate images with noise properties as close as possible to that of the data. However, characterizing the background level in the data is itself a challenging task, let alone the fact that we wish to model the effect of the background noise with just a simple constant Gaussian noise. In this work, we take an approximate approach using SEXTRACTOR quantities and empirically calibrate the noise level instead of deriving it from first principles. We defer a more sophisticated background model to future work.

The basic idea is that the aperture magnitude error versus aperture magnitude relation, for large enough apertures, is only a function of the background noise. Thus, once we know this one-to-one relation as a function of background noise, we could in principle apply the appropriate background noise level to the simulations. In principle, this relation could be derived analytically and the procedure described below is unnecessary. However, since our background model includes a Lanczos resampling, this changes slightly the statistical property of the noise, complicating the relation. In addition, we want to avoid any potential nonlinear processes in SEXTRACTOR that could be missed in the calculation.

Operationally, we calibrate the noise at the $10 \sigma$ galaxy limiting $(2 \operatorname{arcsec})$ aperture magnitude, that is, the $2 \operatorname{arcsec}$ aperture magnitude where the magnitude error is $(2.5 / 10 \ln (10)) \sim 0.1086$. The calibration procedure is described below:

1. Generate UFIG images with the median seeing of the data and a range of different background levels.

2. Run SEXTRACTOR on the simulated images in the same way as on the SV data.

3. Make cuts FLAGS $=0$ and CLASS_STAR $<0.9$ on SEXTRACTOR to get a clean sample of galaxies.

4. Bin the galaxies in MAG_APER_4 bins of 0.01 and find the bin where MAGERR_APER_4 $\sim 0.1086$, this MAG_APER_4 corresponds roughly to the $10 \sigma$ galaxy limiting magnitude.

5. For these simulations, plot the noise level versus 2 arcsec aperture limiting magnitude and fit the relation.
In Figure 11, we show the final derived calibration curve used to convert an desired aperture limiting magnitude to a noise level we input to UFIG. This calibration will change slightly for images with different seeing and source population, but the level of accuracy $(\sim 0.02 \mathrm{mag})$ is sufficient for our purpose here.

\section{REFERENCES}

Agarwal, N., Ho, S., Myers, A. D., et al. 2014, JCAP, 04, 007

Agostinelli, S., Allison, J., Amako, K., et al. 2003, NIMPA, 506, 250

Albrecht, A., Bernstein, G., Cahn, R., et al. 2006, arXiv:astro-ph/0609591

Allen, S. W., Evrard, A. E., \& Mantz, A. B. 2011, ARA\&A, 49, 409

Amara, A., \& Réfrégier, A. 2008, MNRAS, 391, 228

Balbinot, E., Santiago, B., Girardi, L., et al. 2012, in ASP Conf. Ser. 461, Astronomical Data Analysis Software and Systems XXI, ed. P. Ballester, D. Egret, \& N. P. F. Lorente (San Francisco, CA: ASP), 287

Becker, M. R. 2013, MNRAS, 435, 115

Behroozi, P. S., Conroy, C., \& Wechsler, R. H. 2010, ApJ, 717, 379

Bengtsson, H.-U., \& Sjöstrand, T. 1987, CoPhC, 46, 43

Bergé, J., Gamper, L., Réfrégier, A., \& Amara, A. 2013, Astron. Comput., 1, 23 Beringer, J., Arguin, J.-F., Barnett, R. M., et al. 2012, PhRvD, 86, 010001

Bertin, E. 2006, in ASP Conf. Ser. 351, Astronomical Data Analysis Software and Systems XV, ed. C. Gabriel, C. Arviset, D. Ponz, \& S. Enrique (San Francisco, CA: ASP), 112

Bertin, E. 2009, MmSAI, 80, 422

Bertin, E. 2011, in ASP Conf. Ser. 442, Astronomical Data Analysis Software and Systems XX, ed. I. N. Evans, A. Accomazzi, D. J. Mink, \& A. H. Rots (San Francisco, CA: ASP), 435

Bertin, E., \& Arnouts, S. 1996, A\&AS, 117, 393

Bertin, E., Mellier, Y., Radovich, M., et al. 2002, in ASP Conf. Ser. 281, Astronomical Data Analysis Software and Systems XI, ed. D. A. Bohlender, D. Durand, \& T. H. Handley (San Francisco, CA: ASP), 228

Binder, K., \& Heermann, D. W. 2010, Monte Carlo Simulation in Statistical Physics (Berlin: Springer)

Blanton, M. R., Schlegel, D. J., Strauss, M. A., et al. 2005, AJ, 129, 2562

Bouy, H., Bertin, E., Moraux, E., et al. 2013, A\&A, 554, A101

Bridle, S., Balan, S. T., Bethge, M., et al. 2010, MNRAS, 405, 2044

Busha, M. T., Wechsler, R. H., Becker, M. R., Erickson, B., \& Evrard, A. E. 2013, in American Astronomical Society Meeting Abstracts, Vol. 221, 341.07

Connolly, A. J., Peterson, J., Jernigan, J. G., et al. 2010, Proc. SPIE, 7738, 773810

Conroy, C., Wechsler, R. H., \& Kravtsov, A. V. 2006, ApJ, 647, 201

de Jong, J. T. A., Verdoes Kleijn, G. A., Kuijken, K. H., \& Valentijn, E. A. 2013, ExA, 35, 25

Desai, S., Armstrong, R., Mohr, J. J., et al. 2012, ApJ, 757, 83

Diehl, H. T., Abbott, T. M. C., Annis, J., et al. 2014, Proc. SPIE, 9149, 91490V

Diehl, H. T., \& Dark Energy Survey Collaboration. 2012, in American Astronomical Society Meeting Abstracts, Vol. 219, \#413.05

Dietrich, J. P., Werner, N., Clowe, D., et al. 2012, Natur, 487, 202

Duchon, C. E. 1979, JApMe, 18, 1016

Fadely, R., Hogg, D. W., \& Willman, B. 2012, ApJ, 760, 15

Frieman, J. A., Turner, M. S., \& Huterer, D. 2008, ARA\&A, 46, 385

Gerke, B. F., Wechsler, R. H., Behroozi, P. S., et al. 2013, ApJS, 208, 1

Girardi, L., Barbieri, M., Groenewegen, M. A. T., et al. 2012, in TRILEGAL, a TRIdimensional ModeL of thE GALaxy: Status and Future, ed. A. Miglio, J. Montalbán, \& A. Noels (Berlin: Springer), 165

Griffith, R. L., Cooper, M. C., Newman, J. A., et al. 2012, ApJS, 200, 9 
Henrion, M., Mortlock, D. J., Hand, D. J., \& Gandy, A. 2011, MNRAS, 412, 2286

Hilbert, S., Hartlap, J., White, S. D. M., \& Schneider, P. 2009, A\&A, 499, 31

Ho, S., Agarwal, N., Myers, A. D., et al. 2013, arXiv:1311.2597

Hodapp, K. W., Kaiser, N., Aussel, H., et al. 2004, AN, 325, 636

Huff, E. M., \& Graves, G. J. 2014, ApJL, 780, L16

Huterer, D. 2010, GReGr, 42, 2177

Huterer, D., Takada, M., Bernstein, G., \& Jain, B. 2006, MNRAS, 366, 101

Jouvel, S., Kneib, J.-P., Ilbert, O., et al. 2009, A\&A, 504, 359

Kiessling, A., Heavens, A. F., Taylor, A. N., \& Joachimi, B. 2011, MNRAS, 414, 2235

Kitching, T. D., Balan, S. T., Bridle, S., et al. 2012, MNRAS, 423, 3163

Kron, R. G. 1980, ApJS, 43, 305

Leistedt, B., Peiris, H. V., Mortlock, D. J., Benoit-Lévy, A., \& Pontzen, A. 2013, MNRAS, 435, 1857

Lin, H., Kuropatkin, N., Wechsler, R., et al. 2010, BAAS, 42, 470.07

Maddox, N., Hewett, P. C., Péroux, C., Nestor, D. B., \& Wisotzki, L. 2012, MNRAS, 424, 2876

Marchesini, G., Webber, B. R., Abbiendi, G., et al. 1992, CoPhC, 67, 465

Melchior, P., Suchyta, E., Huff, E., et al. 2014, arXiv:1405.4285

Miyazaki, S., Komiyama, Y., Nakaya, H., et al. 2012, Proc. SPIE, 8446, $84460 Z$

Moffat, A. F. J. 1969, A\&A, 3, 455

Mohr, J. J., Armstrong, R., Bertin, E., et al. 2012, Proc. SPIE, 8451, 84510D
Nelson, W. R., \& Namito, Y. 1990, in Presented at the International Conference on Supercomputing in Nuclear Applications, Mito City, Japan, March 12-16, 12

Ngeow, C., Mohr, J. J., Alam, T., et al. 2006, Proc. SPIE, 6270, 23

Peng, C. Y., Ho, L. C., Impey, C. D., \& Rix, H.-W. 2002, AJ, 124, 266

Peterson, J. R., \& Jernigan, J. G. 2013, ascl soft, 1307.011

Pollo, A., Rybka, P., \& Takeuchi, T. T. 2010, A\&A, 514, A3

Riebe, K., Partl, A. M., Enke, H., et al. 2013, AN, 334, 691

Ross, A. J., Percival, W. J., Sánchez, A. G., et al. 2012, MNRAS, 424, 564

Rossetto, B. M., Santiago, B. X., Girardi, L., et al. 2011, AJ, 141, 185

Ruiz-Lapuente, P. 2014, Dark Energy (New York: Cambridge Univ. Press)

Scolnic, D., Rest, A., Riess, A., et al. 2014, ApJ, 795, 45

Sérsic, J. L. 1963, BAAA, 6, 41

Sevilla, I., Armstrong, R., Bertin, E., et al. 2011, arXiv:1109.6741

Smith, B., Sigurdsson, S., \& Abel, T. 2008, MNRAS, 385, 1443

Soumagnac, M. T., Abdalla, F. B., Lahav, O., et al. 2013, arXiv:1306.5236

Springel, V., \& Hernquist, L. 2003, MNRAS, 339, 289

The Dark Energy Survey Collaboration. 2005, arXiv:astro-ph/0510346

Vogelsberger, M., Sijacki, D., Kereš, D., Springel, V., \& Hernquist, L. 2012, MNRAS, 425, 3024

Weinberg, D. H., Mortonson, M. J., Eisenstein, D. J., et al. 2013, PhR, 530, 87 White, M., Tinker, J. L., \& McBride, C. K. 2014, MNRAS, 437, 2594

Zhang, Y., McKay, T. A., Bertin, E., et al. 2014, arXiv:1409.2885 